EOMmun Communication et organisation

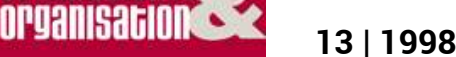

Management par projet et logiques

communicationnelles

\title{
Le management par projet : une logique de communication « imparfaite'
}

Françoise Bernard

\section{(Q) OpenEdition}

\section{Journals}

Édition électronique

URL : http://journals.openedition.org/communicationorganisation/2038

DOI : 10.4000/communicationorganisation.2038

ISSN : 1775-3546

Éditeur

Presses universitaires de Bordeaux

Édition imprimée

Date de publication : 1 mai 1998

ISSN : 1168-5549

Référence électronique

Françoise Bernard, «Le management par projet : une logique de communication « imparfaite' »,

Communication et organisation [En ligne], 13| 1998, mis en ligne le 26 mars 2012, consulté le 30 avril

2019. URL : http://journals.openedition.org/communicationorganisation/2038 ; DOI : 10.4000/

communicationorganisation.2038

Ce document a été généré automatiquement le 30 avril 2019

(c) Presses universitaires de Bordeaux 


\title{
Le management par projet : une logique de communication « imparfaite'
}

\author{
Françoise Bernard
}

1 Le développement des équipes de projet peut être considéré comme étant une des formes de reconfiguration des équipes de travail. Le management par projet peut, quant à lui, être considéré comme conduisant à une forme de reconfiguration organisationnelle, qui n'est cependant pas strictement reliée au mouvement du « reengineering ». L'équipe de projet peut être définie comme étant centrée sur l'action finalisée. Elle participe d'une approche téléologique du travail et de l'organisation. Plus généralement, elle s'inscrit dans le mouvement de "décentralisation», de "débureaucratisassions» et de «déformalisation» des organisations. Dans les organisations du secteur marchand, ce mouvement est issu d'un constat: celui de la différence entre firmes. Cette différence serait fondée non plus sur la capacité de production mais sur «la capacité à innover " dans un environnement marqué par l'incertitude (Alter, 1989, p. 27) mais aussi par les mutations du marché, celui-ci devenant un «marché offreur dans une économie de variété » (Ségrestin, 1996, p. 175).

2 Dans les organisations du secteur social, la conduite de projet est développée pour plus d'efficience sociale. Elle renouvelle certaines pratiques issues de logiques institutionnelles fortes, par exemple celles des organisations syndicales.

3 Notre réflexion s'appuie sur des éléments de comparaison du management et de la communication de projet entre, d'une part des organisations relevant de l'économie de marché et, d'autre part, des organisations relevant de l'économie sociale. Les études de cas que nous avons réalisées dans ces deux environnements, nous ont conduite à identifier des divergences mais aussi des convergences dans le management par projet entre ces deux catégories d'organisation. Nous allons nous intéresser à l'approche comparative du management par projet entre ces deux environnements. Nous allons également analyser les effets de reformulation et de surcodage de la notion de projet provoqués par sa circulation entre un contexte marchand et un contexte social. Ce qui nous conduit à explorer deux hypothèses : celle d'un apprentissage organisationnel lié à 
un apprentissage communicationnel et celle de l'influence de la communication ordinaire dans la coproduction des innovations par les équipes de projet.

\section{Le projet entre communication « institutionnalisante » et communication « artificielle »}

4 Le projet, comme modèle d'action finalisée, circule d'un contexte organisationnel à l'autre. À l'occasion de cette circulation, les boucles de rétroaction sont nombreuses et elles sont autant de points de transfert des reformulations successives du projet issues des expérimentations du management de et par projet dans des contextes différents.

5 En ce qui concerne le contexte de l'économie sociale, nos études sont centrées la réorganisation en structure de projet de la conception et de la réalisation des activités sociales, culturelles et de loisirs pour les salariés d'une très grande entreprise publique française. En ce qui concerne le contexte de l'économie marchande, nos études sont centrées sur le management de projets dans une firme de haute technologie.

6 L'identification des éléments de contextes nécessite de définir à quels types d'organisation nous avons affaire, d'autant plus que l'intimité des concepts d'organisation et de communication est forte (Bernard, 1998 b). En terme de théories des organisations, les organisations du secteur social relèvent plutôt d'une définition de l'organisation «institution», les organisations du secteur de production des hautes technologies relèvent plutôt d'une définition de l'organisation «artefact " (Simon, 1983). La notion d'organisation institution valorise une approche centrée sur les notions de relations réglées et de conventions au détriment de notions souvent individualiste et naturalistes en vigueur dans les autres théories de l'organisation, la théorie classique par exemple. L'organisation «institution» que nous avons pu observer est centrée sur les «éléments de valeur » (Fiol et Solé, 1993); par exemple le projet institutionnel est un réfèrent "éthique » très important qui est censé guider les projets d'activités, il s'agit d'un management par les valeurs. L'organisation « artefact » que nous avons pu observer est centrée sur les " éléments de fait " (Fiol et Solé, 1993); dans ce cas, le management en général et le management par projet en particulier sont perçus comme «ensemble de savoirs et savoir-faire tenus pour neutre » (Fiol et Solé, 1993, p. 88). Dans le cas du management de et par projet, cette «neutralité » des savoirs et savoir-faire dissimule en fait une rupture épistémologique et un changement de présupposés qui peuvent être résumés dans le passage d'une logique «objet " mécaniciste à une logique "projet » complexe. Un tel changement affecte l'organisation elle-même. En effet, le management par projet s'inscrit dans une organisation « artefact » qui est elle-même redéfinie comme un projet: "Il y a artefact lors-qu 'il y a adaptation délibérée d'un projet dans un environnement » (Simon, 1981, p. 9, cité par Le Moigne, 1994, p. 88).

7 Il y aurait donc deux catégories d'organisation et vraisemblablement deux logiques de communication. La première logique peut être qualifiée de communication " institutionnalisante », lorsque par exemple les membres de l'équipe de projet échangent de manière apparemment informelle et que cet informel renvoie de fait mais implicitement à des règles de communication personnelles ou familiales, règles apprises hors champ professionnel. La seconde logique peut être qualifiée de «communication artificielle » (Roqueplo, 1990), dans le sens où tout comme l'intelligence artificielle, elle comporterait une dimension technologique mais aussi stratégique et finalisée. Or, nous 
pensons que la circulation du projet du champ de l'économie marchande vers le champ de l'économie sociale a pour effet de brouiller une telle catégorisation polarisée. Le métissage de catégorisations conduit à des recatégorisations. Par exemple, la communication institutionnalisante (qui, précisons le, ne se confond pas avec la communication institutionnelle managériale) traverse l'ensemble des formes organisationnelles, y compris l'organisation en équipe de projet.

8 En sortant des organisations marchandes, on observe que la notion de projet, explicitement orientée vers l'action et les buts, tend à être en quelque sorte réinstitutionnalisée. L'essaimage du management par projet dans un ensemble d'institutions éducatives, sociales, sportives, etc. n'est pas sans effet rétroactif sur le management par projet dans l'ensemble des environnements - économique et social -. Par exemple: les étudiants apprennent en conduisant des projets mais aussi en construisant un projet universitaire, les demandeurs d'emploi sont censés relancer leur projet professionnel, les associations caritatives, humanitaires fonctionnent également en structure de projet, etc. Le management des projets « d'activités sociales » en rapprochant la finalité des valeurs met en lumière l'inévitable institutionalisation de toute organisation. La logique projet deviendrait donc, par le détour en économie sociale, un modèle d'action finalisée somme toute de plus en plus institutionnalisé.

9 En effet, le projet introduit une approche téléologique dans le management par les « valeurs » et, plus subtilement, le projet en migrant hors du champ du management par les «buts » se charge de valeurs. Pour les organisations du secteur social, le logique projet orienté vers des buts conduit à une remise en cause des logiques institutionnelles plutôt centrées sur la reproduction d'une certaine "tradition». Pour ces organisations, la conduite de projet accélère de fait une forme de métissage entre organisation « institution » (centrés sur les valeurs) et organisation «artefact » (centrée sur les buts finalisés).

10 Le « paradigme » du projet deviendrait, selon une dynamique de boucles récursives, pour toutes les organisations, un paradigme de l'éthique de l'action finalisée. Autrement dit, le projet d'équipe, revisité par l'économie sociale, est un lieu où rationalité téléologique (focalisée sur les buts) et rationalité axiologique (focalisée sur les valeurs) (Weber, 1971) ne s'opposeraient plus. Une telle perspective est une remise en cause du présupposé de séparabilité entre les faits et les valeurs, entre la science et la métaphysique proposé par le positivisme logique dont Herbert Simon se revendique (Fiol et Solé, 1993). Ce qui signifie, par ailleurs, que l'étude de la communication du management par projet passe par l'analyse non seulement de la communication inter-organisations mais également inter-environnements.

11 Le paradigme projet qui relève d'une «intentionnalité artefact» révèle en fait l'impossibilité pour la communication de projet d'échapper, peu ou prou, à certaines formes d'institutionnalisation. D'autant plus que les institutions peuvent être informelles, comme le sont par exemple « les représentations collectives dans le cadre de rapports sociaux donnés » (Clerc, Périsse et Villeval, 1994, p. 181). Ces représentations peuvent influencer les comportements des acteurs. Par exemple, la représentation dominante et mécaniciste de la communication conduit souvent à «linéariser» les processus complexes de la communication, ce qui ralentit les formes d'apprentissage communicationnel de la complexité en équipe de projet. 


\section{Le projet : de l'apprentissage organisationnel à l'apprentissage communicationnel}

12 Dans la mesure où la communication de projet est décalée vis-à-vis de la communication hiérarchique habituelle, l'hypothèse que nous formulons est que la communication d'une équipe de projet passe par des apprentissages communicationnels nouveaux. Les membres de l'équipe de projet ont, en particulier, à définir collectivement les procédures qui vont leur permettre de résoudre des problème, ces problèmes étant les projets.

Dès les années 50 , Herbert Simon suggère d'appliquer la notion d'apprentissage non plus seulement à l'individu mais également à l'organisation. L'apprentissage organisationnel renvoie à l'idée de connaissances collectives propres à l'organisation et non réductibles aux connaissances individuelles (Bazzoli et Bouabdallah, p.174). La théorie de l'apprentissage organisationnel distingue trois niveaux d'apprentissage qui remettent plus ou moins en cause les savoirs établis et mémorisés (Argyris et Schbn, 1978). Le dernier niveau étant un apprentissage par apprentissage. Gérard Koenig note, qu'en trente ans, l'attention s'est déplacée de la gestion « de la répétition » à « l'apprentissage en marchant» (1994, p.76). Le projet est précisément, selon nous, une situation d'apprentissage en marchant. La notion de cognition collective dans un contexte marqué par la contingence, l'irréversibilité et la labilité peut constituer une autre définition du projet. Le projet peut aussi être défini par ce qu'il n'est pas. En particulier, la notion de projet est opposée à la notion d'opération (Koenig) et la logique projet est opposée à la logique objet (Le Moigne, 1994).

14 Notre hypothèse est que le projet comme mode de travail et d'organisation est un lieu d'apprentissage : apprentissage organisationnel nous l'avons dit mais aussi apprentissage communicationnel. Autrement dit, "un apprentissage peut en cacher un autre" (Hatchuel, 1994, p. 112), en l'occurrence l'apprentissage organisationnel dans une équipe de projet est étroitement lié à l'apprentissage communicationnel. Nous allons nous intéresser plus spécifiquement à cette deuxième dimension de l'apprentissage: l'apprentissage communicationnel.

15 L'apprentissage organisationnel a été défini comme "un phénomène collectif d'acquisition et d'élaboration de compétences qui, plus ou moins durablement plus ou moins profondément, modifie la gestion des situations et les situations elles-mêmes » (Koenig, 1994, p. 78). Dans une équipe de projet, l'apprentissage communicationnel peut donc être défini de la manière suivante: "un phénomène collectif d'acquisition et d'élaboration de connaissances et de compétences communicationnelles qui, plus ou moins ponctuellement ou plus ou moins durablement, oriente la gestion des projets et les projets eux-mêmes ». Les connaissances communicationnelles diversifiées sont distribuées dans l'équipe de projet. Elles peuvent être explicitées mais elles sont le plus souvent tacites.

«La connaissance tacite inclut des éléments cognitifs, les schémas, croyances, modèles mentaux... définissent notre vision des choses et des éléments techniques correspondant à un savoir-faire ancré dans des contextes spécifiques» (Nonaka, 1994, cité in Reix, 1995, p. 18).

16 La connaissance communicationnelle collective est étayée par une somme de connaissances individuelles tacites. La constitution d'une équipe de projet est un moment privilégié pour l'explicitation de certaines connaissances communicationnelles qui permettent la régulation du travail en équipe et donc du projet. C'est aussi un temps de 
ré-ajustement des connaissances tacites entre membre de l'équipe, nécessité par le changement de contexte: l'équipe de projet recomposée et provisoire se substitue à l'équipe de travail habituelle et stable.

La rationalité du projet comme composante de la rationalité procédurale de l'organisation, serait donc liée aux jeux et enjeux communicationnels. Ce qui renvoie à l'idée : « d'une métarationalité émergente de nature nécessairement délibérative (au sens de «l'éthique de la discussion» mise en avant par Habermas (Habermas, 1987,1992; Ladrière, 1992) et non plus seulement instrumentale » (Jacot, 1994, p. 208). Autrement dit, l'équipe de projet représente un espace possible d'émergence, dans le filet des normes institutionnelles, organisationnelles et communicationnelles, d'événements communicationnels qui favorisent à leur tour d'autres événements: innovations de production et de service dans et par la conduite de projets. Événements communicationnels en ce sens qu'il ne s'agit pas d'une répétition de normes et de comportements qui renverrait à des savoirs communicationnels mémorisés et à des «routines » relationnelles. Le management par projet participe d'une conception de l'organisation apte au "problem solving ( (centrée sur l'action) mais il peut être, dans certains cas, une préfiguration de ce que serait une organisation apte au "problem designing » (centrée sur «l'intelligence »), c'est-à-dire capable de « se fixer ses objectifs et d'évaluer ses propres processus de raisonnement et de logiques» (Tacot, 1994, p. 208). Or, une telle capacité « d'intelligence collective » passe par des processus interactifs.

L'apprentissage communicationnel émerge de l'ensemble des interactions et des formes de coopération qui se noue à l'occasion de la réalisation de projet. Il s'agit d'une forme d'apprentissage des interactions par les interactions, et, au-delà, de production de connaissances communicationnelles à partir de l'interaction. Les questions de l'explicitation, de la modélisation et de la transférabilité de ces savoirs tacites, non formalisés, sont pertinentes pour développer une culture du projet. Autrement dit, la culture de projet est aussi reliée à la mémorisation des savoirs et savoir-faire communicationnels.

19 Le management par projet est un mode de management de l'innovation qui prend appui "sur les capacités d'initiative et sur les savoirs diffus ", c'est le lieu de valorisation des expériences et approches diverses qui s'éloignent a priori des savoirs reconnus (Mayère, 1994, p. 14). Nous pensons que la mise en commun de ces savoirs diffus passe par la mise en commun de savoir-faire communicationnels qui n'ont que peu de rapports avec la communication manageriale et la communication hiérarchique. Ainsi une nouvelle relation de travail émerge qui correspond à un nouvelle convention définie par le couple " adhésion contre autonomie relative » (Mayère, p. 15).

Plus généralement, les processus d'apprentissage du management par projet s'effectuent aussi par des «apprentissages par interactions » (Lundwall, 1994) entre organisations à l'occasion des formes de coopération interorganisations multiples (sous-traitance, alliances, etc.).

\section{La communication de projet : une communication d'innovation par la communication ordinaire}

21 L'hypothèse que nous explorons plus précisément dans nos études est celle d'une régulation communicationnelle des projets marquée par l'importance de «la 
communication ordinaire » comme composante centrale de la communication de projet. Or, selon nous, la communication "procédurale » de fonctionnement des projets serait indissociable, parce qu'elle émerge de séquences de communication ordinaire, d'une communication «institutionnalisante et sociétale». La communication ordinaire est institutionnalisante car elle est chargée d'implicites qui renvoient à d'autres règles et normes de communication « cristallisées » dans d'autres lieux institutionnels, la famille, l'Université, l'Ecole d'ingénieurs, etc., par exemple.

$\mathrm{Au}$ niveau empirique, nous avons été amenée à observer concrètement les phases de communication formelle de la gestion de projet : réunions hebdomadaires par exemple, ainsi que les phases plus informelles : communication par courrier électronique, échange de face à face ou téléphonique, etc.

L'observation de la dynamique communicationnelle conduit à identifier l'événement fondateur du projet.

24 L'équipe de projet peut être définie comme un «acteur collectif. Dans l'industrie, cet acteur collectif se met en place souvent à partir d'une directive du service marketing. Le chef de projet prend alors l'initiative, acte fondateur du projet, de réunir pour la première fois les acteurs potentiels du projet. En management social, le déclencheur est souvent une orientation du Conseil d'Administration composé d'élus qui est transmise aux «fonctionnels », ceux-ci sont des médiateurs vis-à-vis des opérationnels - bénévoles ou salariés - qui mènent concrètement les activités sur le terrain. Le responsable d'activités prend lui aussi l'initiative, acte fondateur du projet, de réunir les acteurs du projet.

25 Le projet est conduit dans une perspective d'ouverture communicationnelle. Il est accompagné d'une stratégie de communication en direction de partenaires - internes et externes à l'organisation - qui sont classés en fonction de leur degré d'implication dans le projet.

Dans tous les cas (économie sociale et économie marchande), les animateurs de projet que nous avons interviewés se sont retrouvés dans le témoignage de l'un d'entre eux, chef de projet, ingénieur d'une filiale fabriquant des composants électroniques dans une très grande firme française :

«Ce qui représente la difficulté majeure c'est de gérer une véritable communauté motivée par le projet ».

L'explicitation de cette difficulté renvoie à des catégorisations qui sont :

29 - le passage d'une notion de pouvoir à une notion d'autorité,

30 - la motivation,

31 - la gestion de conflits en particulier autour du contrôle de l'information.

32 Nous pouvons noter que les catégorisations proposées par ce chef de projet sont très « académiques » en management, en gestion des ressources humaines et en informationcommunication et que, curieusement, le projet ne conduit pas ou peu à des catégorisations différentes, voire nouvelles. Prenons un exemple: la question de la motivation, celle-ci n'est pas reproblématisée. Or, certains auteurs ont reproblématisé cette question de motivation en question de sens, sens du projet qui renvoie au sens du travail et au sens de la vie (Sievers, 1990). Tout se passe en fait comme si, dans ce témoignage, comme dans bien d'autres témoignages convergeants, la nouveauté de la 
situation de projet était saisie dans des catégorisations antérieures et exogènes à la notion de projet.

Ainsi, des contradictions apparaissent entre présupposés : ceux qui renvoient à une notion d'équipe «objet " (organisée et pilotée de l'extérieur) et ceux qui accompagnent une notion d'équipe « action» (auto-éco-organisatrice (Morin, 1988) par et dans l'action). Dans ce deuxième cas, les présupposés sont aussi ceux d'un projet partagé dans et par une " communauté » de travail. La notion de communauté peut renvoyer à une définition institutionnelle de l'équipe. Cependant, la notion de communauté très polysémique, pour ne pas dire floue, relève du langage analogique de la communication (Watzlawick, 1972). Il est cependant nécessaire de revenir sur la définition de cette notion. Il est intéressant de noter que les sociologues ont plutôt étudié la notion de communauté dans un contexte religieux (Weber, 1971) ou militaire (Shils et Janowitz, 1978). Ainsi, par analogie, l'hypothèse d'un processus de "communalisation» d'une équipe de projet peut être formulée. En ce qui concerne la communalisation d'un groupe religieux, Max Weber avait déjà souligné la double dimension d'organisation et d'institutionnalisation (Weber, 1971). Les membres d'une communauté de projet étant «homophiles» (Lazarsfeld et Merton, 1965), ils sont censés partager des intérêts et des goûts communs. L'homophilie pour les membres de la "communauté de projet " peut être traduite en termes d'intérêts et de goûts communs pour l'action et la cognition collectives, pour la négociation, pour la coopération, pour la symétrie des relations, etc. L'équipe de projet orientée vers l'action et la cognition collectives se distinguerait donc d'une équipe de travail « objet » orientée vers l'application des consignes hiérarchiques. L'observation des équipes de projet montre que la contradiction «objet/projet » traverse en fait les équipes de projet ellesmêmes.

Équipe de projet «objet» ou équipe de projet «action" renvoient également à deux conceptions différentes de la communication: «la communication modèle » et «la communication imparfaite » (Livet, 1998).

Dans la communication modèle, les acteurs du projet sont des décodeurs dans la perspective du schéma canonique de la communication - émetteur/ récepteur de signes -. Dans un tel cas, la communication de projet reste limitée à une définition et à une pratique linéaires de la communication. Dans la communication imparfaite, ces mêmes acteurs sont des interprètes inférentiels (Sperber et Wilson, 1987) - interprètes et coproducteurs d'indices-. La communauté de projet ne préexiste pas à la communication de projet, pas plus que celle-ci ne préexiste pas à la conduite de projet, elle est un processus qui est coproduit en coproduisant l'échange. La communauté des « interacteurs » se construit dans l'interaction. Pierre Livet a montré que le présupposé selon lequel « la communication de face à face assurait la synchronie et donc le savoir commun » relève du présupposé de décodage de la communication modèle. Les échanges de face à face seraient en fait des « suggestions d'inférences à développer » (Livet, 1998, p. 4). S'il peut y avoir des pistes d'inférence différentes entre locuteurs, des limitations à une prolifération de ces pistes sont cependant produites par la dimension institutionnelle de la communication. Dans une équipe de projet, la structure symétrique de la communication fondée sur la primauté des buts partagés et non pas sur la primauté des statuts hiérarchiques favorise des inférences communes aux membres de l'équipe en terme de rapports de confiance et de coopération. Le desserrage des règles et conventions de la communication hiérarchique conduit par ailleurs à des formes d'intrusion plus forte d'autres règles et conventions communicationnelles, par exemple celles de l'institution 
familiale. Un nouveau réajustement des conventions de communication est implicitement effectué par le groupe, il correspond à un métissage inédit de conventions professionnelles et non professionnelles.

Les régulateurs du projet (chefs de projet ou animateurs de projet) sont placés dans une situation communicationnelle inhabituelle en ce sens que, plus qu'ailleurs et que d'habitude, le projet qu'ils animent ne peut aboutir que si, et seulement si, d'autres acteurs s'engagent dans le processus du projet. Cet engagement d'autres acteurs peut être analysé dans le cadre des théories de l'action (Joule et Beauvois, 1987) mais aussi dans le cadre des théories communicationnelles, en particulier, nous l'avons dit, avec la théorie de la communication modèle et la théorie de la «communication imparfaite ». Celle-ci rejoint une approche de la communication définie comme énoncé total prenant en compte le « soma communicationnel » (Bernard, 1998 a).

\section{BIBLIOGRAPHIE}

BERNARD F., «La communication de changements : vers une heuristique de l'induction ", Communication et Organisation, GREC/O, Université Michel de Montaigne, Bordeaux, 1998 a.

BERNARD F., La communication organisationnelle : parcours vers une légitimité scientifique. Contribution épistémologique, une approche par une histoire des idées ", Travaux d'HDR, Grenoble, 1998 b.

BAZZOLI, L., BOUABDALLAH K., « Pour une approche institutionnaliste de l'organisation », in Jacot J.-H. (sous la dir.) Formes anciennes, formes nouvelles d'organisation, Lyon, PUL, 1994, pp. 155-178.

CLERC A., Périsse M., VILLEVAL M.-C, « De l'organisation externalisante à l'organisation interactive, le jeu des institutions dans le changement », in Jacot J.-H. (sous la dir.) Formes anciennes, formes nouvelles d'organisation, Lyon, PUL, 1994, pp. 179-204.

FIOL M., SOLE A., « La question du mangement selon Simon », Revue Française de Gestion, n 94, juin-juil.-août 1993, pp. 87-95.

HATCHUEL A., « Apprentissages collectifs et activités de conception », Revue Française de Gestion, n • 99, juin-juil.-août 1994, pp. 109-120.

JOULE R.V., BEAUVOIS J.L., Petit traité de manipulation à l'usage des honnêtes gens, Grenoble, Presses Universitaires de Grenoble, 1987.

LADRIERE J., Les enjeux de la rationalité, Paris, Aubier-Unesco, 1977.

LAZARFELD P., MERTON R.K., « Friendship as a social process », in Gouldner A.W., Studies in leadership : leadership and democratic action, New York, Russell \& Russell, 1965.

LE MOIGNE J.-L., Le constructivisme, Paris ESF, tomes 1 et 2, 1994,1995.

LE MOIGNE J.-L., « Sur la capacité de la raison à discerner rationalité substantive et rationalité procédurale », Cahiers d'Economie Politique, n² 24-25,1994, pp. 125-159.

LIVET P., De la communication modèle à la communication imparfaite, Conférence invités à l’Université de Lisbonne, 1998. 
LUNDWALL B.A., National Systems of Innovation : Towards a Theory of Innovation and Interactive Learning, London, Pinter Publishers, 1992.

MORIN E., La Méthode, La Connaissance de la Connaissance, 3, Paris, Le Seuil, 1988.

MORIN E., « La complexité, grille de lecture des organisations », Management France, jan/fév 1986.

NONAKA I., «A dynamic theory of organizational knowledge creation », Organization Science, vol. 5, n 1, fév 1994, pp. 14-37.

PERRIN J., « Applications de « l'approche artefact » aux structures organisationnelles », in Jacot J.-H. (sous la dir.) Formes anciennes, formes nouvelles d'organisation, Lyon, PUL, 1994.

REK R., « Savoir tacite et savoir formalisé dans l'entreprise », Revue Française de Gestion, n 105, sept.-oct. 1995, pp. 17-28.

ROQUEPLO P., «Les avatars de la communication du fait de la technique », in Sfez L. et Coutlée G. (sous la dir.), Technologies et symboliques de la communication, Grenoble, PUG, 1990, pp. 404-417.

SHILS E.A. et JANOWITZ M., « Cohésion et désintégration de la Wehrmacht » (trad, franc.) in Mendras, Eléments de sociologie, Textes, Paris, Armand Colin, 1978, pp. 45-79.

SIMON H. A., « De la rationalité substantive à la rationalité procédurale », 1976, (trad, franc.), Pistes, n 3, oct. 1992, pp. 25-43.

SIMON H. A., Models of my Life, Basic Books, 1991.

SIMON H. A., Administration et processus de décision, Paris, Económica, 1983.

SPERBERD., Wilson D., La pertinence, Paris, Ed. de Minuit, 1989.

WATZLAWICK P. et ai, Une logique de la communication, Paris, Le Seuil, 1972.

WEBERM., Economie et Société, Paris, Plon, 1971.

\section{RÉSUMÉS}

L'auteur propose une approche comparative du management par projet dans deux contextes distincts: celui de l'économie sociale et celui de l'économie marchande. L'étude de la communication de projet dans les organisations institution « et dans les organisations « artefact «montre des formes de métissage entre une communication de projet finalisée (centrée sur l'action) et une communication institutionnalisante (centrée sur les valeurs). La circulation des pratiques de projet d'un contexte à l'autre, par le jeu des interactions-interorganisations, favorise des fertilisations et des dynamiques entre approche téléologique et approche institutionnelle. L'équipe de projet est donc un lieu d'apprentissage organisationnel mais aussi celui d'un apprentissage communicationnel par l'interaction. La communication de projet s'éloigne d'une « communication modèle « où les interactants sont définis comme des décodeurs. Elle relèverait plutôt d'une «communication imparfaite «où les interactants sont des « interprètes inférentiels ». L'auteur montre que la communication ordinaire entre les membres des équipes de projet oriente la conduite de projet et le projet lui-même. Autrement dit la communication ordinaire influence les capacités d'innovation des équipes.

The author present a compative approach of project management in two distinctive contexts : social economy and market economy. The study of communication project in «institution « organizations and in « artefact « organizations shows hybrid forms between a finalized project communication (action centered) and an institutionalising communication (value-centered). The 
circulation of project practices from one context to the other through interorganizations intectactions favors fertiflization and dynamics between a technological approach and an institutional approach. The project teams is therefore a locus place of an organizational learning but it is also a place for a communicational learning through interaction. Project communication differs from communication models (classical models) in which interacting persons are defined as decoders. It is closer to an imperfect communication where interacting persons are infering interpreters. This paper questions discusses the concept of a « culture project « as resulting from the memory of communicational knowledge and praxis.. In all cases $C$ artefact organazitions and « institution « organizations) the author shows that ordinary and daily communication between project team members guides project behavior and project per se. In other words ordinary communication influences teams innonvation capacités and abilities.

\section{AUTEUR}

\section{FRANÇOISE BERNARD}

Françoise Bernard, Maître de conférences, habilitée à diriger des recherches en sciences de l'information et de la communication, enseigne à l'Université de Provence (Aix-

Marseille 1). Auteur de publications, articles et études spécialisés en communication des organisations, elle est membre fondateur du groupe national de recherches et d'études en communication des organisations de la SFSIC, elle est aussi membre du groupe national de recherches et d'études « Théories et Pratiques Scientifiques « de la SFSIC. Chargée de Mission Communication auprès du Président d'Université, elle est également responsable de la presse de l'Université de Provence (version papier, version électronique). 